\title{
Effect of Mirror Therapy on Motor Function in Extremities and Daily Activities in Stroke Patients
}

\author{
Rakad Al-masaeed ${ }^{1}$, Mohamed ALBashtawy ${ }^{2}$, Nahla Al Ali ${ }^{3}$, Khitam Ibrahem Mohammad ${ }^{4}$, \\ Bayan Albashtawy $^{5}$, Fadi Fawares ${ }^{6}$, Abdullah Alkhawaldeh ${ }^{7}$ \\ ${ }^{1} R N$, Master Student, Critical Care Nursing, Princess Salma Faculty of Nursing, AL al-Bayt University, Mafraq, \\ Jordan, ${ }^{2}$ Professor, Princess Salma Faculty of Nursing, AL al-Bayt University, Mafraq, Jordan, ${ }^{3}$ Associate \\ Professor, Community and Mental Health Nursing, Faculty of Nursing, Jordan University of Science \& Technology, \\ Jordan, ${ }^{4}$ Associate professor, Department of Midwifery, Faculty of Nursing, University of Science \& Technology, \\ Jordan, ${ }^{5}$ Medical Intern, King Abdullah University Hospital, Jordan, ${ }^{6}$ Nurse Educator at King Hussein Cancer \\ Center, Amman, Jordan, ${ }^{7}$ Assistance Professor, Princess Salma Faculty of Nursing, AL al-Bayt University, Mafraq,
} Jordan

\begin{abstract}
Stroke is disease cause of partial loss some of brain functions that can lead to many of disorders, such as motor, perception, language, sensory, and can lead to limitation in daily activities. Mirror therapy (MT) is a new therapeutic method used for stroke patients. This short review was intended to assess the effect of the MT on motor function in extremities and daily activities in stroke patients. The electronic searching was conducted in Google Scholar and PubMed. this review paper confirmed that that apply of MT on stroke patients give benefits and significant improve the some of motor function and some of daily activities. Further studies are essential to verify MT long-term effects and its impact on daily activities among stroke patients.
\end{abstract}

Keywords: Stroke, Mirror Therapy, Motor Function, Daily Activities

\section{Introduction}

Stroke is a disease that cause of partial loss some of brain function, and may lead to many disorders, such as motor, perception, language, and sensory. In addition, stroke can lead to limitation in daily activities, such as eating, drinking, and dressing ${ }^{(1)}$. Stroke is two types: Ischemic (88\%) and hemorrhagic $(12 \%)^{(2)}$.

Stroke occur between 2.7 - 4.7 per 1000 people, and one of three disease cause of death, and one-third of the patient's development of permanent disabilities (3). More than $60 \%$ of stroke patients have impaired of daily

\section{Corresponding Author:}

Abdullah Alkhawaldeh, $\mathrm{PhD}$, Assistance Professor, Princess Salma Faculty of Nursing, AL al-Bayt University, Mafraq, Jordan.

Email: dr-abd@aabu.edu.jo, abdo_752012@yahoo.com activities related to neurological deficits ${ }^{(4)}$. Hemiplegia is paralysis in one side of body occurs in one upper extremity and one lower extremity occurs from stroke (5).

Rehabilitation play very important role in the recovery of patients after stroke, one of these methods is Mirror Therapy (MT). The MT is a new therapeutic method use for patients who have hemiparesis or motor disabilities occur from stroke, this method shows positive outcomes with those patients ${ }^{(3)}$. It applies by place the unaffected limb front the mirror and the affected limb back the mirror, then the movement of unaffected limb give for patient illusion this occur in affected limb ${ }^{(6)}$. The MT was used with stroke patients because it is low cost, easy to use, and can be applied at patient's home ${ }^{(7)}$.

Many people have inadequate information about the benefits of MT. The current study may add to knowledge about this type of therapy, and so to improve movement in affected limb and help patients to take care 
of themselves. This short review paper was intended to assess the effect of the MT on motor function in extremities and daily activities in stroke patients.

\section{Method}

Search methods: The electronic searching was conducted in a different database: Google Scholar and PubMed. Key search terms used: Stroke, Mirror Therapy, Motor Function, Daily Activities.

The studies that focused on the topic of MT were published in English between 2015 and 2020 were included in the review. While, studies published before 2015 were excluded.

Search outcome: Exploring of literature yield about 35 studies for review, after reading the studies about 25 study were excluded and 10 studies met the inclusion criteria.

\section{Discussion}

It is uncertain exactly how MT works, it's been proposed that this illusion causes brain changes that assistance the patient improve his mobility. The MT is comparatively easy to do, and has the possible to be accomplished at individual household ${ }^{(8)}$.

The MT was used to increase motor function after stroke. Many studies found the MT improve motor function and daily activities in stroke patients. A study aimed to examine the effects of MT together with exercise tasks on the function of the upper limbs and activities of daily living. The study finding confirmed that MT is more effective comparing with conventional therapy for the training of stroke patients to develop their upper limb function and activities of daily living ${ }^{(9)}$.

Also, the MT with the conventional stroke rehabilitation program was examined. A study explored the effects of mirror therapy on spasticity, pain intensity and upper limb motor functions among patients with hemiplegia accompanied by complex regional pain syndrome type 1 . The study found that adding of MT to a conventional stroke rehabilitation program provides more progress in the upper limb motor functions and pain perception than conventional therapy alone ${ }^{(10)}$. Another recent study found that the use of MT in stroke patients have significant improve the level of motor function ${ }^{(2)}$.
Past studies found evidence of a significant effect of MT on motor function compared with other interventions. Besides, there was evidence of a significant effect of MT for walking velocity, passive range of motion for ankle dorsiflexion, step length and balance capacity ${ }^{(11)}$. Another study found that the use of MT in rehabilitation treatment for stroke patients can improve of lower limb motor function ${ }^{(12) .}$

Finally, there was similarly some confirmation that MT may decrease pain. However, the level of evidence is inadequate, and further investigation is required to evaluate the influence of MT on pain ${ }^{(13)}$.

\section{Conclusion}

These short review shows the MT is safe and effective and it has benefits in improve some of the motor functions in extremities and daily activities in stroke patients. This short review paper provides some information about the benefits of MT to improve motor function and daily activities in stroke patients. Further studies are necessary to verify MT long-term effects and its impact on daily activities among patients in different stage of stroke.

Conflict of Interest: No conflict of interest.

Ethical Clearance: Taken from Princess Salma Faculty of Nursing, AL al-Bayt University ethical committee.

\section{Source of Funding: Self.}

\section{References}

1. Bailey, Ryan R. Lifestyle modification for secondary stroke prevention. American journal of lifestyle medicine 2018; 12:140-147.

2. Varghese, Brigin Ann; NISHA, C. Margret; EVENCY, A. Reena. Effectiveness of Mirror Therapy on Motor Function among Patients with Cerebro Vascular Accident. Amarjeet Kaur Sandhu 2020; 12:227.†

3. Shabaani Mehr, Maryam, et al. The effect of mirror therapy on the walking ability of patients after stroke. Journal of Holistic Nursing and Midwifery 2019; 29:200-209.'

4. Bhoraniya, Sirajahemad H., Daxa G. Mishra, and Shweta M. Parikh. "The effect of mirror therapy on 

the gait of chronic stroke patients: A randomized controlled trial." National Journal of Physiology, Pharmacy and Pharmacology 2018; 8:1321-1325.

5. Chinnavan, Elanchezhian, Rishikesavan Ragupathy, and Yu Chye Wah. "Effectiveness of Mirror Therapy on Upper Limb Motor Functions Among Hemiplegic Patients.” Bangladesh Journal of Medical Science 2020; 19:208-213.†

6. Chiluba, B. C., et al. "A Review on the Effects of Mirror Therapy in Stroke Patients with Partial Paralysis." Journal of Integral Sciences 2019; 2:1923.†

7. de Oliveira, Pedro, Glória Maria Andrade do Couto, and Maria Gorete Reis. "Mirror therapy and self-care autonomy after stroke: an intervention program." Revista de Enfermagem Referência 2018; 4:95-105A.

8. Thieme, Holm, et al. "Mirror therapy for improving motor function after stroke." Cochrane Database of Systematic Reviews 2018; 7.

9. Kim, Kyunghoon, et al. "Effects of mirror therapy combined with motor tasks on upper extremity function and activities daily living of stroke patients." Journal of physical therapy science 2016; 28:483-487.

110. Vural, Secil Pervane, et al. "Effects of mirror therapy in stroke patients with complex regional pain syndrome type 1: a randomized controlled study." Archives of physical medicine and rehabilitation 2016; 97:575-581.9

11. P. Broderick, F. Horgan, C. Blake, M. Ehrensberger, D. Simpson, K. Monaghan. Mirror therapy for improving lower limb motor function and mobility after stroke: A systematic review and meta-analysis. Gait \& Posture 2018; 63:208-220.

12. Wang, Haiyan, et al. "Effect and mechanism of mirror therapy on rehabilitation of lower limb motor function in patients with stroke hemiplegia". An International Journal of Medical Sciences 2017; 28.

13. Barbin, Jessie, et al. "The effects of mirror therapy on pain and motor control of phantom limb in amputees: a systematic review." Annals of physical and rehabilitation medicine 2016; 59:270-275.' 